\title{
Superior Colliculus Inactivation Causes Stable Offsets in Eye Position during Tracking
}

\author{
Ziad M. Hafed, ${ }^{1}$ Laurent Goffart, ${ }^{2}$ and Richard J. Krauzlis ${ }^{1}$ \\ ${ }^{1}$ Systems Neurobiology Laboratory, Salk Institute for Biological Studies, La Jolla, California 92037, and ${ }^{2}$ Institut de Neurosciences Cognitives de la \\ Méditerranée, Equipe Dynamique de la Perception Visuelle et de l'Action, Unité Mixte de Recherche 6193, Centre National de la Recherche Scientifique, \\ Aix-Marseille Universités, 13402 Marseille, France
}

The primate superior colliculus (SC) is often viewed as composed of two distinct motor zones with complementary functions: a peripheral region that helps generate saccades to eccentric targets and a central one that maintains fixation by suppressing saccades. Here, we directly tested the alternative interpretation that topography in the SC is not strictly motor, nor does it form two distinct zones, but instead forms a single map of behaviorally relevant goal locations. Primates tracked the invisible midpoint between two moving stimuli, such that the stimuli guiding tracking were peripheral whereas the inferred movement goal was foveal and parafoveal. Temporary inactivation of neurons in the central portion of the topographic map of the SC, representing the invisible goal, caused stable offsets in eye position during tracking that were directed away from the retinotopic position encoded by the inactivated SC site. Critically, these offsets were not accompanied by a systematic inability to generate or suppress saccades, and they were not fully explained by motor deficits in saccades, smooth pursuit, or fixation. In addition, the magnitude of the offset depended on the eccentricity of the inactivated site as well as the degree of spatial uncertainty associated with the behavioral goal. These results indicate that gaze control depends on the balance of activity across a map of goal locations in the SC, and that by silencing some of the neurons in the normally active population representing the behavioral goal, focal inactivation causes a biased estimate of where to look.

Key words: superior colliculus; pursuit; voluntary eye movement; stimulus-response; reversible inactivation; muscimol

\section{Introduction}

The primate superior colliculus (SC) has long been viewed as a motor map for saccades, with neurons in the intermediate and deep layers of this structure forming a topographic representation of saccade vectors (Robinson, 1972; Schiller and Stryker, 1972; Wurtz and Albano, 1980). Subsequent studies have also identified fixation-related activity in the portion of the SC corresponding to the fovea, suggesting that the SC contains two distinct zones issuing competing motor commands for maintaining fixation or generating saccades (Munoz and Guitton, 1991; Munoz and Wurtz, 1993a,b). However, an alternative interpretation holds that the SC contains a single, continuous map of behaviorally relevant goal locations (Krauzlis et al., 1997, 2004). Consistent with this view, the so-called fixation neurons of the SC are modulated during small saccades and smooth pursuit, and this modulation depends on both the location of the target (Basso et al., 2000; Krauzlis et al., 1997, 2000) as well as its behavioral relevance (Krauzlis and Dill, 2002; Krauzlis, 2003).

\footnotetext{
Received March 27, 2008; revised July 3, 2008; accepted July 7, 2008.

This work was supported by the Natural Sciences and Engineering Research Council of Canada, the Sloan-Swartz Center for Theoretical Neurobiology, and National Institutes of Health (NIH) Grant EY12212 (Z.M.H.), the Agence Nationale de la Recherche REflex Tricks in Natural and Artificial Eyes grant (L.G.), and NIH Grant EY12212 (R.J.K.). We are grateful for the feedback of C. Wehrhahn on a previous version of this manuscript and for the help of Natalie Dill in the experiments.

Correspondence should be addressed to Ziad M. Hafed, Systems Neurobiology Laboratory, Salk Institute for Biological Studies, 10010 North Torrey Pines Road, La Jolla, CA 92037. E-mail: zhafed@salk.edu. DOI:10.1523/JNEUROSCI.1317-08.2008

Copyright $\odot 2008$ Society for Neuroscience $\quad$ 0270-6474/08/288124-14\$15.00/0
}

Despite this evidence, there have been few direct tests of the goal location interpretation. Reversible inactivation of the SC affects saccade target selection (McPeek and Keller, 2004), consistent with this interpretation. In addition, subthreshold microstimulation of the SC biases target selection for both pursuit and saccades, and the pursuit results show that the effect is based on the location of the target rather than the direction of eye movement (Carello and Krauzlis, 2004). These results demonstrate that the SC is involved in resolving competition among possible visual targets, not just possible saccades, but they do not distinguish between goal location and visual stimulus location.

The hypothesis that the SC contains a continuous map of goal locations is also challenged by anatomical data (Büttner-Ennever et al., 1999; Takahashi et al., 2005) often viewed as evidence for a "fixation zone" in the SC. Specifically, the SC projects to omnipause neurons (OPN), whose activity presumably prevents saccades (Keller, 1974; Evinger et al., 1982; Keller and Edelman, 1994; Everling et al., 1998), and the strength of these projections is greatest from the foveal representation of the rostral SC (Gandhi and Keller, 1997; Büttner-Ennever et al., 1999). If the rostral SC does not issue fixation commands, what is the function of these projections?

Here, we tested the goal location hypothesis using a tracking task that dissociated the movement goal from the visual stimuli (see Fig. 1). When we recorded SC activity during such a task, we found that it represents the location of the goal rather than the visual features, so our main objective in the present experiments 
Table 1. List of the locations in the visual field that were represented by our injected SC sites across all experiments

\begin{tabular}{|c|c|c|c|c|}
\hline & $\begin{array}{l}\text { Horizontal eccentricity } \\
\text { (in degrees) }\end{array}$ & $\begin{array}{l}\text { Vertical eccentricity } \\
\text { (in degrees) }\end{array}$ & $\begin{array}{l}\text { Radial eccentricity } \\
\text { (in degrees) }\end{array}$ & $\begin{array}{l}\text { Polar direction (counterclockwise } \\
\text { degrees from right horizontal) }\end{array}$ \\
\hline \multirow[t]{3}{*}{ Right SC } & -0.95 & 1.62 & 1.88 & $120^{a}$ \\
\hline & -2.76 & 2.63 & 3.8 & 136 \\
\hline & -4.11 & -1.78 & 4.49 & 203 \\
\hline \multirow[t]{12}{*}{ Left SC } & 0.5 & -0.56 & 0.75 & 312 \\
\hline & 0.41 & 1.3 & 1.37 & 73 \\
\hline & 2 & -1 & 2.24 & 333 \\
\hline & 2 & 1 & 2.24 & 27 \\
\hline & 2.51 & 2.37 & 3.45 & 43 \\
\hline & 4.5 & -2.5 & 5.1 & $336^{a}$ \\
\hline & 4.57 & 2.47 & 5.2 & 28 \\
\hline & 5.5 & -0.5 & 5.52 & 355 \\
\hline & 5.5 & 1 & 5.59 & 10 \\
\hline & 4.04 & 4.39 & 5.96 & 47 \\
\hline & 6.5 & 2.5 & 6.96 & 21 \\
\hline & 9.5 & 11 & 14.5 & 49 \\
\hline
\end{tabular}

The sites are sorted by brain hemisphere as well as by their retinotopic location and polar coordinates, as estimated from the maps of visually guided saccade latencies after muscimol injection (see Materials and Methods).

${ }^{a}$ The site was also used later (after all muscimol experiments were completed) for a saline control injection.

was to use reversible inactivation to understand the functional significance of this activity. Consistent with the goal location hypothesis, inactivation caused stable eye-position offsets that were not explained by motor deficits. In addition, although the SC projections to OPNs have been assumed to act en masse to suppress saccades, our results suggest that a more complex computation is involved: namely, that the motor decision to generate a corrective movement away from the current gaze location depends on the balance of activity across the population of SC outputs.

\section{Materials and Methods}

Our experiments were controlled by a computer using the Tempo software package (Reflective Computing), and a second computer running the Psychophysics Toolbox in Matlab (MathWorks) (Brainard, 1997; Pelli, 1997) acted as a server device for presenting the visual stimuli. Stimuli were presented with a video monitor $\left(75 \mathrm{~Hz}, \sim 20\right.$ pixels $\left./{ }^{\circ}\right)$ at a viewing distance of $41 \mathrm{~cm}$. Eye movements were recorded using scleral search coils (Judge et al., 1980) and the electromagnetic induction technique (Fuchs and Robinson, 1966) using standard phase detector circuits (Riverbend Instruments). All data and events related to the onset of stimuli were stored on disk during the experiment ( $1 \mathrm{kHz}$ sampling rate) for additional analysis.

Animal preparation. We collected data from two (W and A) adult rhesus monkeys (Macaca mulatta) that were 10 years of age and weighed $12-15 \mathrm{~kg}$. One of the monkeys (W) was used (several weeks before the inactivation experiments) in recording experiments describing the activity of SC neurons during our main behavioral task.

All monkeys were prepared using standard surgical techniques that have been described in detail previously (Krauzlis, 2003), and all experimental protocols for the monkeys were approved by the Institutional Animal Care and Use Committee and complied with United States Public Health Service policy on the humane care and use of laboratory animals.

Behavioral tasks. Our main behavioral paradigm consisted of an "extrafoveal tracking" task, as outlined in Figure 1. In this task, our monkeys were presented with a display consisting of a small white spot over a uniform gray background. Once they fixated this spot, an experimental trial was initiated, and the spot disappeared while two white bars appeared simultaneously at symmetrically opposite locations (see Fig. 1). The bars were oriented perpendicular to the axis connecting their centers to the display center, and they moved sinusoidally together (as one rigid object) along this axis (radial amplitude, $4^{\circ}$; frequency, $0.71 \mathrm{~Hz}$; duration, $3500 \mathrm{~ms}$; starting phase, $0^{\circ}$ or $\left.180^{\circ}\right)$. Bar separation $\left(22\right.$ to $\left.25^{\circ}\right)$ and the motion axis orientation $\left(-45\right.$ to $+60^{\circ}$ from horizontal) depended on the SC site visited (see below). Bar dimensions (in degrees of visual angle) were scaled with their separation as follows: height $=(3.6 / 16) \times$ separation; width $=(1 / 28) \times$ separation.

Three additional tracking conditions were also presented in separate blocks. In each block, the monkey's task was to track an explicit foveal stimulus having an identical motion trajectory as in the extrafoveal tracking condition (see Fig. $6 \mathrm{~A}$ ). This foveal stimulus was alternately either a small white spot $\left(\sim 0.03^{\circ}\right.$ radius $)$, a medium blurred spot (white circle of $1^{\circ}$ radius with Gaussian-blurred edges), or a large blurred spot (white circle of $2^{\circ}$ radius with Gaussian-blurred edges). Thus, for a given SC site, we used a single axis of motion but presented four different visual stimuli. For the medium and large targets, we used circular stimuli with smooth, blurred edges to discourage the monkeys from tracking particular corners or edges and instead induce them to track the entire stimulus as one extended object. This caused the larger targets to have larger spatial uncertainty about the location to track than the smaller ones, which we confirmed by measuring the monkeys' variance in eye position during tracking (see Results).

The monkeys were allowed an initial grace period $(750 \mathrm{~ms})$ to initiate their tracking, during which no spatial constraints on eye position were enforced. After the grace period, the monkeys were required to track each moving stimulus within a set eccentricity from its center (for details, see below, Reversible inactivation). For the extrafoveal tracking condition, the monkeys were trained to infer the invisible midpoint between the two bars and track it as well as they could. Thus, the movement goal in this condition was invisible. We collected 35-50 trials for each motion starting phase, resulting in 70-100 trials per eye-movement condition per experiment.

Other behavioral tasks were used to identify and confirm inactivated sites in the SC. These were standard saccade tasks, which are described briefly below.

Reversible inactivation. We inactivated portions of the SC of monkeys W and A using local muscimol injections (Hikosaka and Wurtz, 1985a,b; Chen et al., 2001) $(0.5 \mu \mathrm{l}, 5 \mu \mathrm{g} / \mu \mathrm{l})$. Our injections were aimed at the intermediate and deep layers of the SC (1.8-2.5 mm below surface) and spanned a range of SC sites in 15 experiments (Table 1). Two additional experiments were excluded from any analysis because muscimol, although it affected the SC, had also spread rostral of the SC (into the pretectum) and caused horizontal nystagmus-like eye movements (Mustari and Fuchs, 1990; Schiff et al., 1990; Munoz and Wurtz, 1993b) in addition to offsets in eye position like those described in this study. We also injected sterile saline solution in two control sessions (one per monkey) at sites inactivated previously with muscimol.

We identified our inactivated sites (Table 1) as follows. The day before each inactivation session, we identified a target site and depth within the SC using single-unit recording and electrical microstimulation to evoke saccades consistent with the metrics encoded by the visited site (microstimulation parameters: $400 \mathrm{~ms}, 500 \mathrm{~Hz},<30 \mu \mathrm{A}$, biphasic pulses). During the inactivation session, we confirmed our site by observing multiunit or single-unit saccade-related activity and/or by evoking saccades with microstimulation. Successful microstimulation was our criterion to proceed with the inactivation experiment. Finally, after injecting muscimol, using a custom-made apparatus modified from Chen et al. (2001), we verified our site by observing latency increases during a visually guided saccade task (Hikosaka and Wurtz, 1985a,b). These latency increases were localized to the region of retinotopic space affected by our injection (see Figs. 3C, $8 \mathrm{~A}$ ), and we used the location with the largest saccade latency as our quantitative estimate of the inactivated SC site (although some of our analyses considered the entire spatial extent of inactivation) (see Figs. 9-11). Injection of the entire volume of muscimol or saline was done over a period of $\sim 30-40 \mathrm{~min}$. 
We ran one round of tracking trials before inactivation (baseline) and one after. Our baseline data set was collected with our injection apparatus resting $\sim 1 \mathrm{~mm}$ or more above our estimate of SC surface obtained from the previous day's recording session. This was done to avoid the possibility of leakage of some muscimol into the SC while we were collecting baseline data. Also, for both the baseline and inactivation data sets, all experimental parameters were identical, although we rewarded the monkeys more generously as the session progressed to keep them motivated. We also typically started with the small target and progressed toward our largest one (extrafoveal tracking) in the baseline set but reversed the order in the inactivation set. This was done to ramp the monkeys down toward an easy task at the end of a long session. Because extrafoveal tracking gave the largest inactivation-induced effects (see Results), this also allowed us to ensure that these large effects were not simply attributable to the fact that muscimol had been in the SC for a long period. Finally, we chose the stimulus motion axis to cross (as closely as possible) the location encoded at the inactivated SC site, although we had one experiment in which we ran the additional control of an orthogonal motion axis (see Fig. 8).

On the next day after each experiment, we collected recovery data with the same stimulus and behavioral parameters as in the inactivation (data not shown). In addition to confirming that the monkeys recovered, we did this to confirm stationarity in the monkeys' baseline tracking behavior (i.e., that changes observed with inactivation were not caused by natural variability in behavior, especially during extrafoveal tracking with an invisible goal).

For the extrafoveal tracking condition, bar separations were $22-25^{\circ}$, meaning that one bar was placed at an eccentricity of $11-12.5^{\circ}$ in the contralateral visual field and the other bar was placed at a similar eccentricity in the ipsilateral visual field. This, in turn, implies that our inactivated sites (Table 1, see Fig. $4 B$ ) were all more rostral (i.e., representing less eccentric locations) than the contralateral bar location (except for one caudal injection made for comparison). In addition, for both the baseline and inactivation trials, our monkeys were required to track the inferred movement goal (midpoint between the bars) to within $4-5^{\circ}$ (which was less stringent than in the small, medium, and large target conditions). Although this range of eye-windowing might seem to be slightly lenient (especially because our monkeys could perform extrafoveal tracking with higher accuracy), it was important to maintain identical eye-monitoring parameters both before and after inactivation. Indeed, because inactivation caused large eye-position offsets (see Results), this necessitated more lax eye monitoring parameters to avoid unfairly penalizing the monkeys. As a result, similar parameters were used in the baseline condition as well.

Data analysis. Eye movements were sampled at $1 \mathrm{kHz}$. Saccades were detected using velocity and acceleration thresholds (Krauzlis and Miles, 1996). During tracking, these thresholds were applied relative to average eye velocity/acceleration to avoid erroneously flagging smooth eye movements (with nonzero velocity and/or acceleration) as saccades (de Brouwer et al., 2002a). All detected saccades were manually verified. We also characterized steady-state pursuit gain by fitting rectified sinusoids to the monkeys' radial eye velocities and comparing their amplitudes to the target velocity amplitude.

To analyze our inactivation results, we plotted eye-position traces before and after inactivation as well as the millisecond-by-millisecond probability of observing catch-up saccades (see Fig. $3 B$ ). We then quantified eye-position offsets caused by inactivation. During sustained tracking $(t>750 \mathrm{~ms})$, we computed the average (desaccaded) eye position relative to the center of the tracked stimulus for each trial (see Fig. 3D, background dots). We then computed the average and SD across trials. Significant differences between baseline and inactivation eye positions (i.e., offsets) were assessed using a multivariate ANOVA (two groups: baseline and inactivation, with two dimensions: horizontal and vertical position). For visualization, we also obtained the magnitude and direction of the offset (inactivation data set relative to baseline data set) and plotted them (see Fig. 4). Estimates of the SEM for these variables were obtained by bootstrapping. We then made similar measurements for a $200 \mathrm{~ms}$ interval centered on $t=0 \mathrm{~ms}$, which allowed us to quantify eye-position offsets during the initial fixation period at trial onset.
We also performed several analyses on catch-up saccades and smooth pursuit gain. For the latter, we compared steady-state gain before and after inactivation. We also measured open-loop eye velocity (i.e., velocity within the first $100 \mathrm{~ms}$ of pursuit) in two different but related ways. First, we measured pursuit onset latency using a "hinge" model like that proposed in (Adler et al., 2002). We then defined a $50 \mathrm{~ms}$ interval starting 50 ms after pursuit onset, during which we computed average saccade-free radial eye velocity before and after inactivation. This was similar to the procedure used in (Basso et al., 2000) to study the effects of SC inactivation on smooth pursuit initiation. Second, we defined a $50 \mathrm{~ms}$ interval starting $125 \mathrm{~ms}$ after trial onset and computed average saccade-free radial eye velocity during this interval before and after inactivation. This interval was chosen to be within the open-loop period of smooth pursuit (pursuit onset occurred at $95 \pm 26 \mathrm{~ms} \mathrm{SD}$ ), and to end before any pursuit direction reversals (which occurred because of the predictable target trajectory in our experiments) and saccades.

As for catch-up saccades, we measured the frequency of these movements during sustained tracking by binning them according to their horizontal and vertical amplitudes (see Fig. 3 E, F). Saccades were clustered around the tracking axis and could be divided into contralesional (saccades contralateral to the injected side, i.e., toward the region encoded by the inactivated neurons) and ipsilesional groups (Fig. 3, compare $C$ and $E, F)$. We summarized catch-up saccade data for all injections by computing the fraction of these saccades that was contralesional before and after inactivation (see Fig. $5 A$ ). We also analyzed inactivationinduced changes in the amplitude and direction of saccades as well as the latencies of the first saccade after trial onset. We defined this first saccade as a pursuit initiation saccade if it had an average latency $<500 \mathrm{~ms}$ from trial onset in the baseline condition. Otherwise, it was not included in this analysis. This procedure was adopted because, in extrafoveal tracking, our monkeys often initiated tracking smoothly without any saccades. In those cases, it was not appropriate to treat the first occurring saccade as being triggered by trial onset.

Modeling. Before performing our inactivation experiments, we characterized SC activity during extrafoveal tracking by recording from single neurons in monkey W, as well as a third monkey not used for the current inactivation experiments. These recording data, which motivated our study and which we only present here in summary form (for purposes of brevity and clarity), allowed us to develop a simple data-driven model of the effects of SC inactivation on extrafoveal tracking. Specifically, by measuring the responses of 117 neurons having a range of preferred eccentricities, we had enough neuronal data to estimate the spatial extent of the activity representing the inferred movement goal during sustained tracking. We did so by binning cells according to their preferred eccentricity, and then computing the average response in each bin when the center of the extrafoveal tracking stimulus (midpoint between the two bars) was at a center-of-gaze position during tracking. Thus, this analysis was equivalent to taking a "snapshot" of SC activity across the map when the goal was centered on the fovea. We then fit these averages with a Gaussian function of the following form:

$$
y=\alpha+\beta \exp \left(-E^{2} / \sigma^{2}\right)
$$

where $E$ is the preferred eccentricity of the neuron, $y$ is the average firing rate of neurons at this eccentricity, $\beta$ is a scaling parameter, and $\sigma$ and $\alpha$ describe the spatial spread and asymptote of SC activity, respectively. After normalizing the output function to a range of $0-1$, our fits for the recording data of Figure $9 A$ (top) gave $\alpha=0.09, \beta=0.91$, and $\sigma=4.7^{\circ}$. We then obtained a two-dimensional (2D) version of this function by assuming a circularly symmetric spatial profile of activity describing the location of the inferred movement goal. This assumption was a reasonable one, because our experiments gave qualitatively similar results across a wide range of neuronal preferred directions and stimulus axes of motion. For visualization purposes, we remapped this function (up to $\pm 20^{\circ}$ in either horizontal or vertical directions) onto anatomical SC coordinates using the following formula (Ottes et al., 1986; Quaia et al., 1998):

$x=B_{x} \ln \left(\operatorname{sqrt}\left(R^{2}+2 A R \operatorname{Cos}(\theta)+A^{2}\right) / A\right)$,

$$
y=B_{y} \operatorname{atan}(R \sin (\theta) /(R \cos (\theta)+A)),
$$


Retinal stimulus

Inferred movement goal: foveal/parafoveal

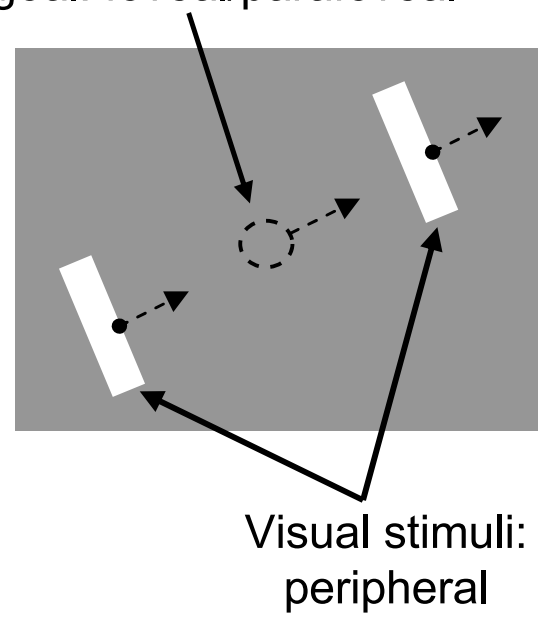

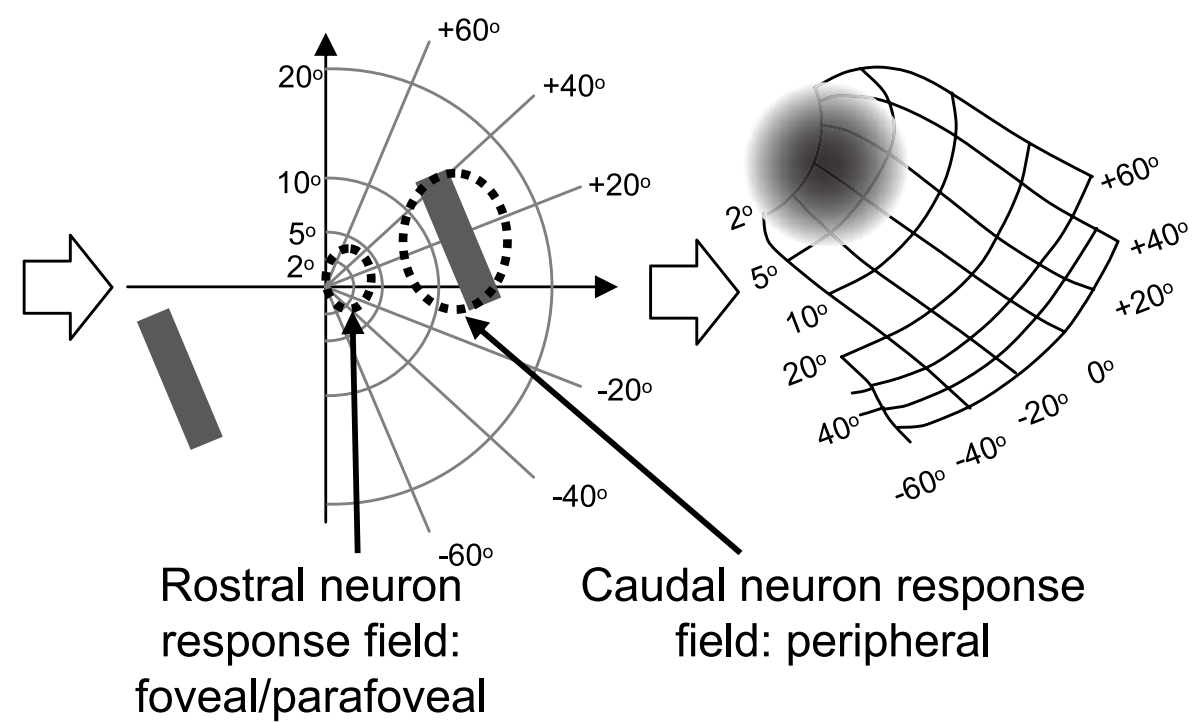

Figure 1. The role of the SC in extrafoveal tracking. Our monkeys viewed two peripheral bars that moved sinusoidally together along an axis orthogonal to the bars' orientation. The monkeys had to track the invisible midpoint between the two bars as it moved (left). With proper tracking, the inferred movement goal was always near the fovea, but the visual stimuli were always peripheral (middle). Thus, neurons in the rostral SC, representing the central visual field, did not have a visual stimulus inside their response fields, but they did represent locations associated with the goal of tracking. Neurons in the caudal SC, representing more peripheral locations, did not represent the goal of tracking. We hypothesized that rostral neurons dominate SC activity during this task despite the lack of a visual stimulus (right, schematic activity is shown as a gray cloud on a classic representation of the SC topographic map).

where $R, \theta$ are the polar coordinates of visual space and $x, y$ are the anatomical SC coordinates. The parameters $A, B_{x}$, and $B_{y}$ were chosen to be $3,1.4$, and 1.8, respectively (Quaia et al., 1998). An example of this transformation can be seen in Figure $9 A$ (bottom).

To simulate the effects of inactivation, we scaled the estimated activity profile obtained above with a "suppression function" describing the spatial extent of muscimol spread. This function was obtained by measuring visually guided saccade latencies in each experiment to identify the inactivated site (see procedure above). After inactivation, latencies increased for some locations but not others, providing an estimate of which SC neurons were affected and to what extent (see Figs. 3C, 8A, 9B) (Hikosaka and Wurtz, 1985a,b). Specifically, we normalized each latency map for each experiment such that it ranged from 0 (for the minimum latency) to 1 (for the peak latency). To match the grid range used to describe neuronal activity above, we also filled an entire representation of $-20-20^{\circ}$ horizontally and vertically by extending the most eccentric measurement outward (using a nearest-neighbor interpolation routine). We then multiplied the modeled SC activity level at each spatial location in the two-dimensional version of Equation 1 above by a suppression factor that was equal to the following:

$$
1-k \times \text { normalized latency, }
$$

where $k$ was a free parameter, which was constant for the entire map. The above equation means the following: the bigger the postinactivation saccade latency for a target at a particular retinal location, the bigger the suppression of SC activity that was assumed by the model. The parameter $k$ was optimized for each experiment to minimize the distance between the experimentally induced eye-position offset (which was the effect of inactivation, as we describe in Results) and the offset obtained by the model. The latter was defined as the offset for which the retinal position of the movement goal (which moved the center of the 2D version of Eq. 1 away from zero) was such that it gave rise to balanced activity across the two SCs (after applying the effects of the suppression function).

\section{Results}

We designed a tracking task that exploits the known ability of humans (Wyatt et al., 1994) and monkeys (Ilg and Thier, 1999) to track objects with "imaginary" foveal targets. In this extrafoveal tracking task, our monkeys were presented with two peripheral bars on either side of the display, and they had to infer the invisible midpoint between these two bars and track it for several seconds (Fig. 1). Thus, during ongoing tracking, the invisible movement goal occupied foveal and parafoveal retinotopic locations. According to the goal location hypothesis of SC function, this means that SC activity during this task should have remained largely rostral, despite the lack of a foveal visual stimulus and despite the fact that the task involved a smooth eye movement output that was distinct from either saccades or fixation.

We first confirmed the above prediction to motivate our inactivation experiments, and we did this by recording from a large population of SC neurons while monkeys performed our task. Although the detailed results of such recordings are not included here for brevity (Hafed and Krauzlis, unpublished observations), sample neurons highlighting the most salient aspects of these results are shown in Figure 2. For the neuron labeled "caudal" in Figure 2, the response field was peripheral. This allowed us to configure our stimulus such that one of the two bars was inside the response field of the neuron but the inferred movement goal (invisible midpoint between the two bars) was not. For the neuron labeled "rostral" in Figure 2, the response field of the neuron covered more central retinotopic locations. This allowed us to have the inferred movement goal (but not the peripheral bars) pass through the response field of the neuron. The modulation in activity during extrafoveal tracking was larger for the rostral neuron than for the caudal neuron. This modulation, which also persisted during periods of smooth tracking in the absence of catch-up saccades, reflected the fact that the inferred movement goal was sweeping in and out of the response field of the rostral neuron as the monkey was trying to actively follow it. Thus, during extrafoveal tracking, the rostral neuron provided a representation of the inferred movement goal, whereas the caudal neuron 
did not provide a similar representation for the stimulus' constituent visual features. Our objective in the present experiments was to test the functional significance of such correlative activity by temporarily perturbing it while the monkeys performed our task.

\section{Reversible inactivation caused stable eye-position offsets during tracking} We reversibly inactivated, using muscimol injections, focal regions in the rostral SC and evaluated the monkeys' ability to perform extrafoveal tracking (Fig. 3A). Figure $3 B$ shows eye-position traces before and after inactivation of a sample SC site that was normally active for $\sim 0.95^{\circ}$ leftward/ $\sim 1.65^{\circ}$ upward saccades (Fig. $3 C$ ). The striking feature of these traces is that the monkey was still able to track, but it did so with a substantial and stable offset in eye position. We quantified this offset by comparing the average eye position during extrafoveal tracking before and after SC inactivation, and we found that inactivation in this sample experiment shifted the eye position by $0.23^{\circ}$ rightward and $1.63^{\circ}$ downward relative to baseline (Fig. 3D). Thus, the induced offset was diametrically opposite the retinotopic location encoded by the inactivated SC site.

Importantly, the inactivation-induced offset in eye position during this sample experiment was not attributable to an impairment in saccade generation, as might be expected if the SC were strictly a motor map for saccades. Inspection of the traces in Figure 3B (bottom) shows that the occurrence of small saccades during sustained tracking was largely unaffected in terms of both absolute frequency as well as absolute onset time. We documented this point further by measuring the distribution of saccades generated during tracking before (Fig. $3 E$ ) and after inactivation (Fig. $3 F$ ). Before inactivation, there was a tendency for more frequent contralateral saccades ( $69 \%$ of all saccades). This tendency remained (66\%; $p>0.05$, binomial distribution) after inactivation, although the programming of these saccades would normally involve activity of SC neurons at the inactivated site. Conversely, we did not observe an increase in the frequency of saccades, as might be expected if our inactivation had impaired the motor control of fixation (Munoz and Guitton, 1991; Munoz and Wurtz, 1993a,b). Thus, the offset in eye position during extrafoveal tracking that we observed in this sample experiment did not reflect a pure motor deficit in either the generation or the suppression of saccades.

We performed a total of 15 reversible inactivation experiments (Table 1). For each, we measured the direction (Fig. 4A) and magnitude (Fig. $4 B$ ) of the eye-position offset induced during tracking by inactivation. All such offsets were significant $(p<$ 0.001, multivariate ANOVA). Moreover, eye position was always shifted in a direction opposite to that encoded at the inactivated site in the SC map (Fig. 4A). The magnitude of this shift was large for a range of inactivated sites covering locations in and around the central visual field (Fig. $4 B$ ). This trend agrees well with
B
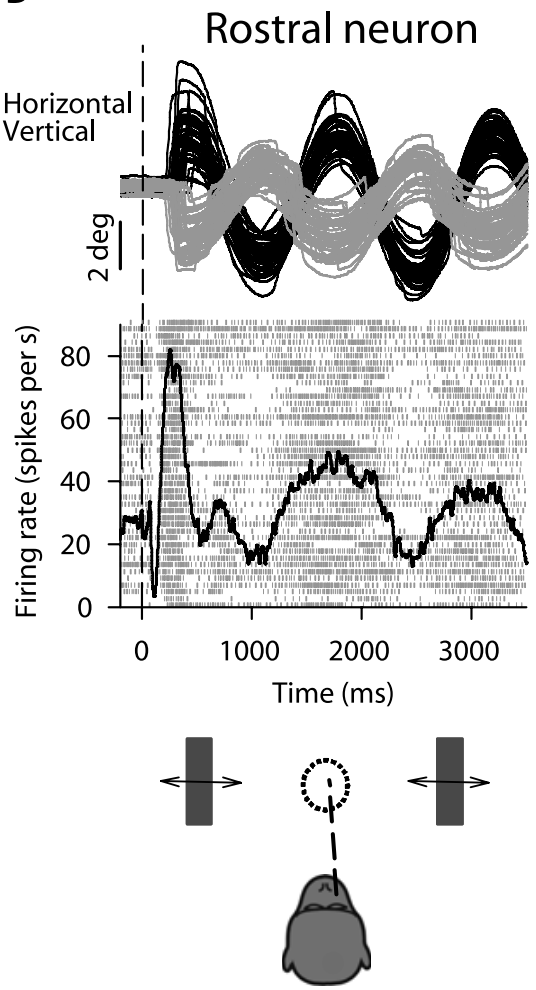

Caudal neuron
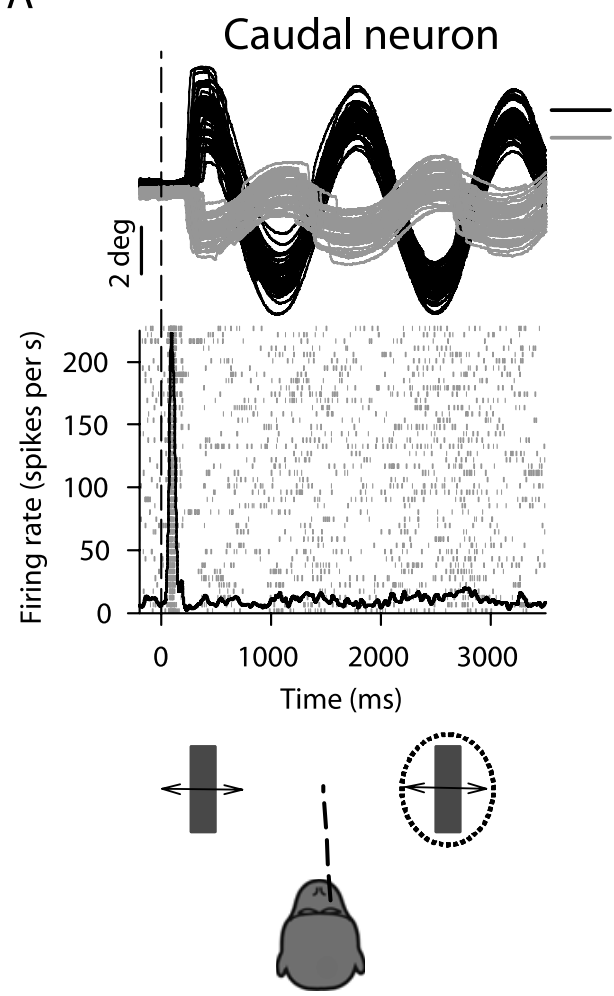

Figure 2. Sample neurons recorded while monkeys performed extrafoveal tracking. $\boldsymbol{A}$, Eye-position traces and responses of a (bottom schematic with dashed oval illustrating the RF). After an initial transient, this neuron was not significantly modulated even though the visual stimulus guiding tracking was inside its RF. $B$, A rostral neuron that reflecting the changing location of the movement goal. Upward deflections in eye-position traces indicate right and up. Neurona responses are shown as individual trial spike rasters as well as average spike density functions.

single-neuron recording data that we obtained from the extrafoveal tracking task and that we summarize in the context of Figures 9-11. For our most eccentric injection, the observed offset was significantly smaller, again suggesting that the population of active neurons in our task did not extend to peripheral locations associated with the features of the visual stimulus.

\section{The offsets were not explained by pure saccade or smooth pursuit deficits}

In our sample inactivation experiment described by Figure 3, we saw no strong evidence that the inactivation-induced offset in eye position during extrafoveal tracking was caused by a deficit in the ability to generate or suppress catch-up saccades. That is, the monkey was generally still able to track and make saccades toward and away from the affected region. We also confirmed that this was true across our experiments. Specifically, for each inactivation experiment, we measured the likelihood that a catch-up saccade occurring during sustained tracking was contralesional (Fig. 5A). Because saccades were predominantly along the tracking axis (Fig. $3 E, F$ ), this measure effectively documented the frequencies of saccades that were directed toward the location encoded at the inactivated site. Only 3 of 15 experiments showed a significant reduction in the monkeys' tendency to generate such saccades, suggesting that the activity that we eliminated by injecting muscimol was not purely saccade related. In all of our experiments, we also never saw irrepressible saccades to locations outside those affected by inactivation (for example, to one of the 
A

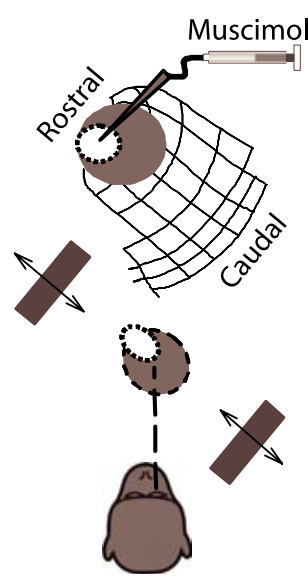

C

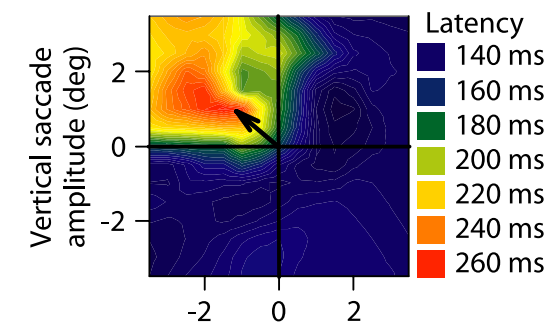

Horizontal saccade amplitude (deg)

E

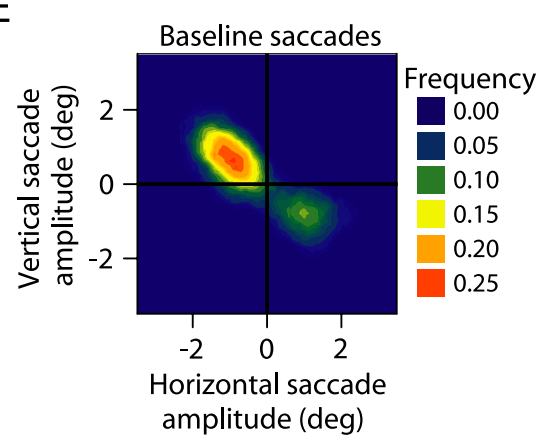

B
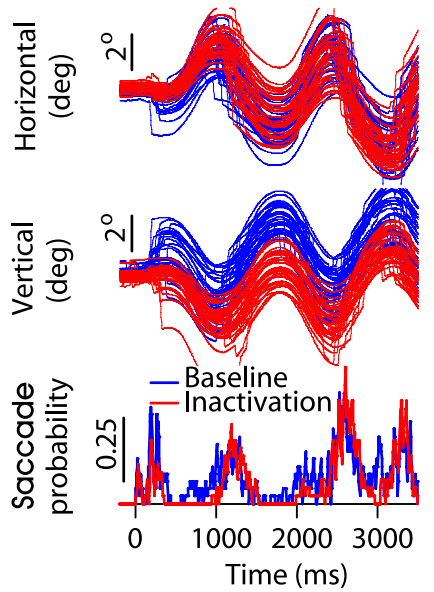

$\mathrm{D}$

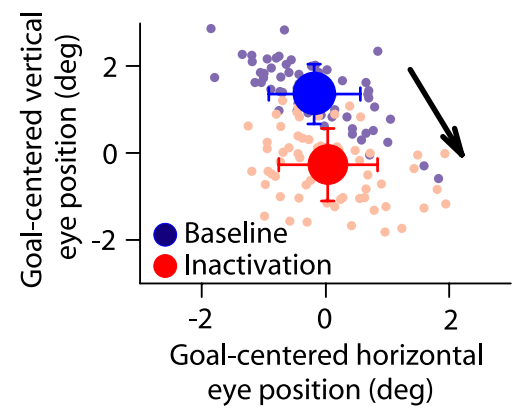

$\mathrm{F}$

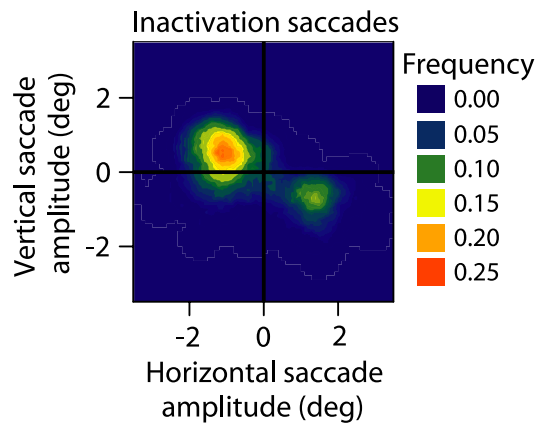

Figure 3. Sample experiment demonstrating the effects of focal inactivation on extrafoveal tracking. $A$, The monkey tracked along a $-45^{\circ}$ axis; we inactivated locations around $-0.95^{\circ}, 1.65^{\circ}(x, y) . B$, Individual-trial eye positions before (blue) and after (red) inactivation. Upward deflections denote right and up. The bottom shows the saccade probability in the traces above. C, We confirmed that we inactivated top left quadrant locations by plotting the latencies of visually guided saccades after muscimol injection and observing localized increases for upward/leftward small saccades when compared with other saccades (Hikosaka and Wurtz, 1985a,b). $\boldsymbol{D}$, Average eye positions relative to the midpoint between the bars (the movement goal) from individual tracking trials before (light blue) and after (light red) inactivation reveal a down/right offset from baseline (arrow). Red and blue dots and error bars denote the mean and SD across trials. $\boldsymbol{E}, \boldsymbol{F}$, Frequency histograms of saccade endpoints occurring during sustained tracking before $(\boldsymbol{E})$ and after $(\boldsymbol{F})$ inactivation suggest that contralesional saccades were not eliminated.

peripheral bars), as might be expected if activity in the rostral SC was necessary for preventing saccades from occurring.

We also considered the possibility that muscimol inactivation might have changed saccade amplitudes and directions enough to explain our observed eye-position offsets. However, Figure $5 B$ shows that this was not the case. In Figure 5B, we computed the net difference between the change in average contralesional and ipsilesional catch-up saccade metrics and plotted it, for each experiment, alongside the observed eye-position offset. As can be seen, inactivation caused minimal effects on catch-up saccade amplitudes and directions during ongoing tracking when compared with its effects on eye position.
It may also be argued that the observed eye-position offsets were simply a result of increased latencies for generating contralesional catch-up saccades (Hikosaka and Wurtz, 1985a,b). Such increased latencies, if any, would introduce a temporal "position lag" during tracking that can manifest itself spatially as a "position offset." Our sample inactivation experiment suggests that this is unlikely, because there were minimal latency effects on saccades, when compared with the effects on eye position (Fig. 3B). Specifically, during ongoing extrafoveal tracking, the onset times of catch-up saccades were nearly identical before and after inactivation (Fig. 3B, bottom). This was probably because of the predictable nature of our target trajectories, but it nonetheless means that the large offsets in eye position that we observed during extrafoveal tracking could not have arisen because of a "universal" increase in all contralesional saccade latencies. Having said that, we further analyzed the effects of inactivation on saccade latencies. Specifically, we plotted the average latency of the first contralesional initiation saccade before and after inactivation (Fig. $5 C)$. We chose the first saccade because its latency was well defined (it was triggered by trial onset), as opposed to later catch-up saccades for which latency is only an inferred parameter (de Brouwer et al., 2002b). As can be seen, Figure $5 C$ plots data from only five experiments rather than 15 (in the remaining sessions, our monkeys resorted to smoothly initiating pursuit). For these five experiments, there was indeed a trend for an increased latency of the first contralesional saccade as a result of inactivation ( 4 of 5 showed significant increases, $p<0.05$, rank-sum test). This is consistent with the known effects of muscimol (Hikosaka and Wurtz, 1985a,b). However, this increased latency was again insufficient to explain the eyeposition offsets. First, our trials (and observed offsets) persisted for several seconds, suggesting that there was plenty of time in every trial for the monkeys to generate a (delayed) saccade and correct for the offsets, and perhaps do that in conjunction with corrections made by the smooth tracking system itself. More importantly, because our task involved sinusoidal trajectories in which tracking direction reversed periodically, an offset that was caused by a pure position lag (in time) is expected to have changed its direction every time the tracking direction changed (the difference between two sinusoids with different temporal lags is a sinusoid). The direction of our observed offsets clearly did not show such a dependence on tracking direction (for two sample inactivation experiments demonstrating this, see Fig. $3 B$, supplemental Movie 1 , available at www.jneurosci.org as supplemental material).

Through a set of simulated tests, we also determined that pure 
A



B

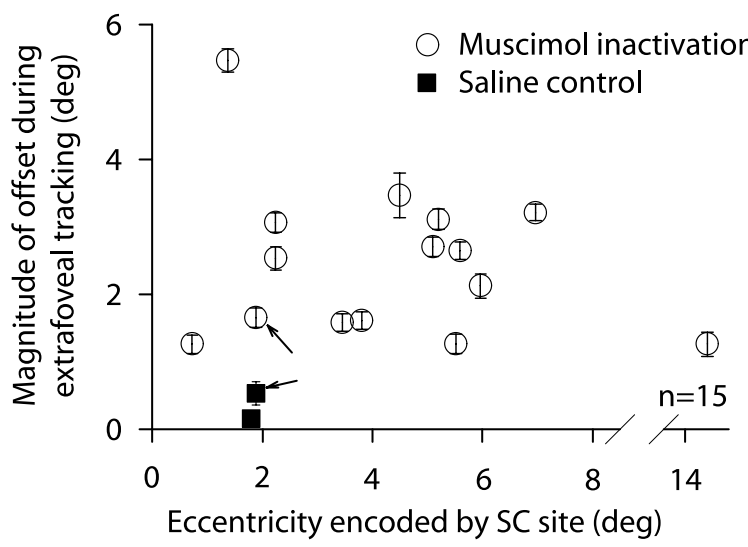

Figure 4. Summary of the effects of $\mathrm{SC}$ inactivation across experiments. A, All inactivations (circles) caused eye-position offsets during tracking that were directed away from the inactivated site (slope of dashed regression line not different from unity; $p>0.05 ; r^{2}=0.9335$ ). Saline injections (squares) did not. $\boldsymbol{B}$, Large offsets were observed for inactivated sites covering a range of eccentricities in and around the central visual field, but not for our most eccentric site representing locations near the peripheral bars defining the stimulus. Error bars denote SEM, and arrows point to the sample experiment of Figure 3 and a saline control experiment at the same SC site.

saccade changes alone were insufficient to explain our experimental data. This idea is illustrated schematically in supplemental Figure 1 (available at www.jneurosci.org as supplemental material). In this figure, we created "simulated" sinusoidal eyeposition traces that tested the effects of three types of pure inactivation-induced saccade changes: increased contralesional catch-up saccade latencies, decreased contralesional catch-up saccade amplitudes, and reduced probability of occurrence of contralesional catch-up saccades. Even in these extreme scenarios, which permitted no other corrections by the oculomotor system, we found that the differences between simulated "baseline" and "inactivation" traces were qualitatively distinct from those that we observed experimentally. Most strikingly, the bottom histogram of saccade onset times in the simulated traces (supplemental Fig. 1, bottom, available at www.jneurosci.org as supplemental material) shows clear temporal shifts between baseline and inactivation that were not observed in our data (Fig. $3 B$, bottom).

Finally, the stable eye-position offsets that we observed after SC inactivation could not be explained by changes in either openloop or steady-state smooth pursuit gain. In terms of open-loop
A

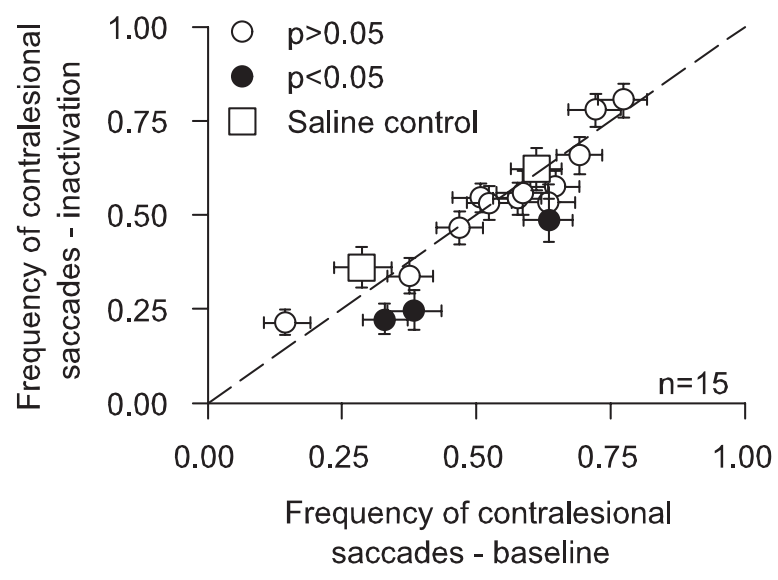

B



Horizontal component (deg)

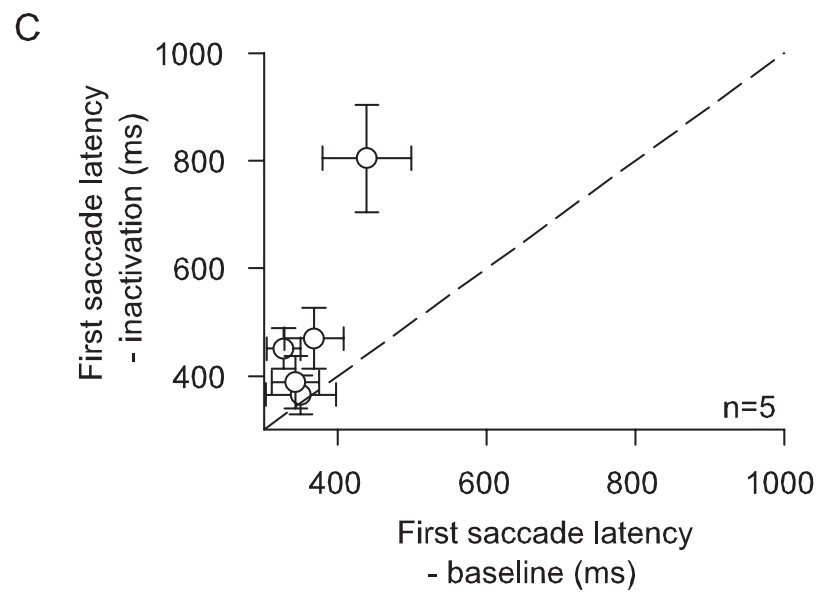

Figure 5. The offsets were not explained by inactivation-induced changes in catch-up saccades. $\boldsymbol{A}$, We plotted the frequency of contralesional saccades before and after inactivation (similar to the analysis of Fig. $3 E, F$ ). Only three experiments showed a reduction in the relative frequency of contralesional catch-up saccades (filled symbols, $p<0.05$, binomial distribution). The saline results (squares) were indistinguishable from the muscimol results (circles). $\boldsymbol{B}$, Eyeposition offsets were also not accounted for by inactivation-induced changes in catch-up saccade amplitude and direction. We compared the horizontal and vertical components of the measured offset to the horizontal and vertical components of the offset expected based on the net changes in catch-up saccade amplitude and direction. Each connected pair of symbols shows one experiment (circle, observed offset; cross, predicted offset based on changes in catch-up saccade metrics). The observed offsets were always larger than those expected based on net changes in catch-up saccade metrics. $C$, The latency of the first initiation saccade (for the contralesional starting phase of tracking) was increased for four of five experiments involving initiation of tracking with a saccade. Error bars denote $95 \%$ confidence intervals $(\boldsymbol{A})$ or SEM (C). 
gain, our monkeys typically initiated their extrafoveal tracking with a small increase in radial eye velocity in the direction of the stimulus motion before quickly reversing directions (or generating a saccade) in anticipation of the reversal of stimulus trajectory. This is illustrated for a representative experiment in supplemental Figure 2 (available at www.jneurosci.org as supplemental material). We analyzed this initial eye velocity before and after inactivation in a manner identical to that in the study of Basso et al. (2000) and also with a slightly different interval that was more tailored to our eye velocity data (described in Materials and Methods). Using both measures, we found no difference across experiments between the initial pursuit response during baseline and inactivation trials. Moreover, this was the case for both contralesional and ipsilesional pursuit initiation. A minority of individual experiments ( 3 of 15 ) showed a small but significant change in open-loop contralesional pursuit initiation gain using the second measure, but this change was an increase rather than a decrease in two of these experiments, an observation that cannot explain our ipsilesional eye-position offsets.

As for steady-state pursuit gain, when we computed the inactivation-induced change in such gain during extrafoveal tracking, we found an average change of $0.01 \pm 0.01$ SEM across sessions. This was not significantly different from zero $(p=0.62$, $t$ test), as expected from our sample inactivation experiment (Fig. $3 B)$.

Therefore, our results suggest that the SC activity that we eliminated by muscimol injection was not primarily involved in driving saccades, fixation, or smooth pursuit. Instead, these results suggest that the inferred movement goal during extrafoveal tracking was represented by a distributed population of rostral SC neurons; by silencing a subset of the normally active neurons, focal inactivation caused an estimate of the goal location that was biased away from the inactivated site.

\section{The magnitude of the offsets depended on the size of the tracked stimulus}

If the goal location interpretation of SC function is correct, then the magnitude of the inactivation-induced eye-position offset should not only depend on the inactivated SC site (Fig. 4B) but also on the size of the normally active population of neurons needed to support the behavior. Consider, for example, an SC site coding for retinotopic locations at $5^{\circ}$ of eccentricity. If most of the activity supporting tracking was more rostral than this site, then inactivation of the site should have minimal effects on behavior. However, the same inactivation should have a significant effect on behavior if the neurons at this site were normally much more involved in supporting it.

To test this idea, we asked our monkeys to track foveal targets of different sizes, in addition to performing extrafoveal tracking (Fig. 6A). For a given inactivation experiment, we observed a clear dependence of inactivation-induced offset magnitude on the size of the tracked target. This is illustrated for a sample session in Figure $6 B$. For this session, our inactivation affected neurons coding for $\sim 5^{\circ}$ of eccentricity. This inactivation had very small effects on the tracking of a small spot (small target). This might be expected because it is known that smooth pursuit of small spots recruits the most rostral of SC sites (Krauzlis et al., 1997, 2000). However, as the size of the target increased, the effect of the same inactivation became progressively larger and larger. In the limit, we observed the largest offset for the extrafoveal tracking condition having an invisible movement goal. This increase in offset magnitude with target size suggests an associated increase in the spatial spread of activity normally needed to rep- resent the movement goal. Our baseline behavioral tracking data support this conclusion. Specifically, even without inactivation, the spatial variance of eye position during tracking became progressively larger with larger target sizes. Figure $6 C$ illustrates this for the same sample experiment of Figure $6 \mathrm{~B}$. To obtain this figure, we computed the average goal-centered eye position in every trial and then computed the SD of this position across trials. This SD clearly increased with target size, suggesting a wider underlying distribution of activity describing the target location.

We found similar results for all 13 experiments in which we ran a full set of target sizes in addition to extrafoveal tracking. These results are summarized in Figure 7, which highlights the effects of both SC site and target size on the magnitude of eyeposition offset caused by inactivation. Specifically, the figure shows that the greatest increase in offset magnitude with target size occurred for parafoveal sites, and the smallest increase occurred for far foveal and far peripheral sites. This is expected because inactivation sites close to either the center or far tails of the active population of neurons are expected to have smaller effects than ones in between (Lee et al., 1988), regardless of population size.

The results of Figure 7 also explain another aspect of our extrafoveal tracking data that can be seen in Figure $3 B$. Specifically, the eye-position offset observed at trial onset in the experiment of Figure $3 B$, although still evident, was much smaller in magnitude than the offset observed during ongoing extrafoveal tracking. This is consistent with the results shown in Figures 6 and 7 , because our extrafoveal tracking trials started with the monkeys fixating a small spot that was identical in size to our "small" target of Figure $6 \mathrm{~A}$. In fact, when we measured the eyeposition offsets at trial onset during all extrafoveal tracking experiments, we found an average magnitude of $0.23 \pm 0.1^{\circ}$ (SD) across sessions. This value was not different from the average offset magnitude observed during tracking of a small target shown in Figure 7, which was $0.32 \pm 0.32^{\circ}(\mathrm{SD})(p>0.05$, rank-sum test and $t$ test).

As for the directions of the observed offsets in the small, medium, and large target conditions, these were also ipsilesional just like for extrafoveal tracking. In fact, we found that the direction of the offset did not depend on the tracking direction. In one of our experiments, we ran an additional control condition in which the tracking axis was orthogonal to the SC site affected by inactivation. Specifically, this inactivation affected neurons representing locations in the lower right quadrant, as can be seen from the map of visually guided saccade latencies shown in Figure $8 \mathrm{~A}$. However, we asked the monkey to perform extrafoveal tracking and "large target" tracking along an axis of motion passing through the top right quadrant (Fig. 8, white arrow). Consistent with our previous results, the observed offsets in eye position were opposite the inactivated site (in direction), and they were larger (in magnitude) for extrafoveal tracking than for the large target (Fig. $8 B, C)$.

Finally, we analyzed catch-up saccades, first saccade latencies, and smooth pursuit gain changes for our small, medium, and large targets just like we did for extrafoveal tracking. The results of these analyses, which were all consistent with those of extrafoveal tracking, are included in supplemental Figures 3-5 (available at www.jneurosci.org as supplemental material) for the sake of completeness. In terms of smooth pursuit initiation (supplemental Fig. 5, top two panels, available at www.jneurosci.org as supplemental material), note that there were more experiments with significant reductions in contralesional pursuit initiation with the small, medium, and large targets than in the extrafoveal track- 


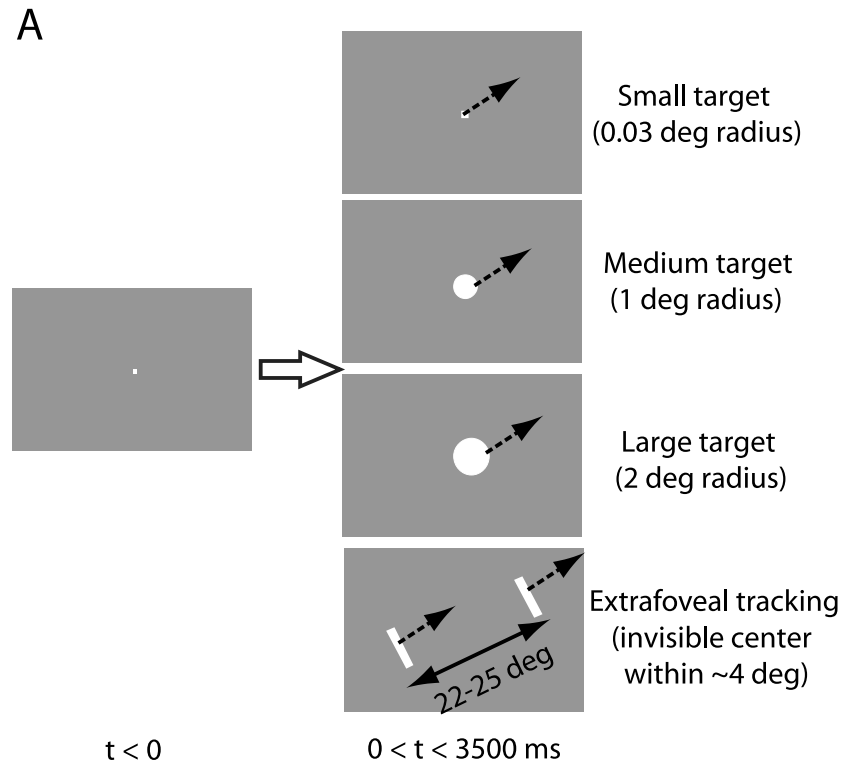

B

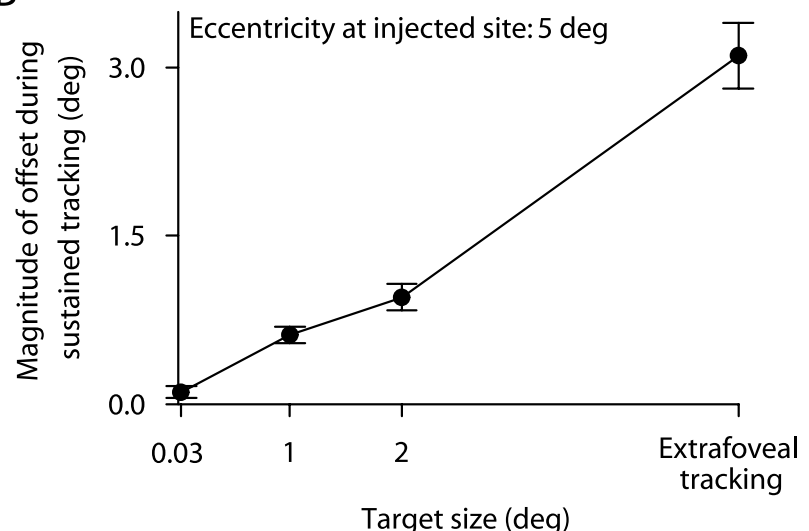

C

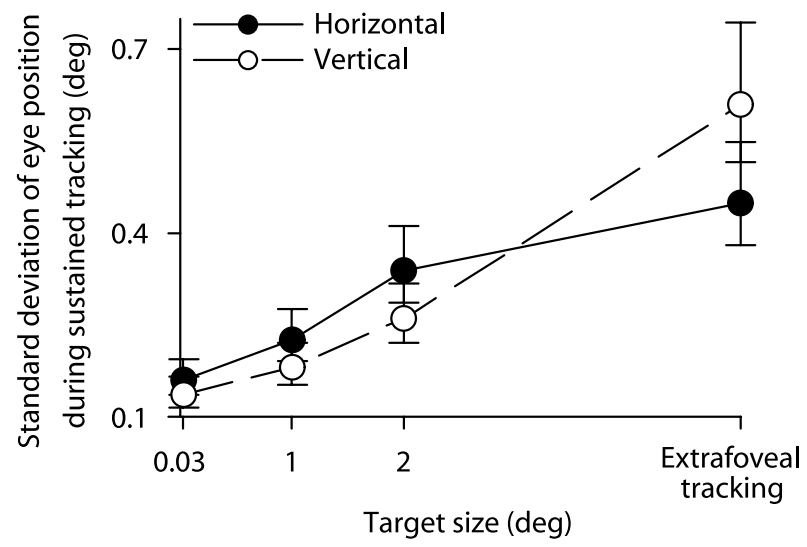

Figure 6. Eye-position offsets were also observed during tracking of explicit foveal targets of different sizes. $\boldsymbol{A}$, In addition to extrafoveal tracking, our monkeys tracked three other targets that had an identical motion trajectory as in extrafoveal tracking. $\boldsymbol{B}$, The inactivation-induced eye-position offset during tracking was observed for all target sizes in the shown sample experiment. The inactivated SC site coded for a $5^{\circ}$ eccentricity. Inactivation of this site caused a small offset when the movement goal was defined by a small spot, but the magnitude of this offset increased with target size. C, We found that larger targets were associated with increased spatial uncertainty about goal location. For the baseline data of the same experiment, we computed the average goal-centered eye position in every trial and plotted the SD across trials for each target size. Larger targets gave rise to more variable eye position, suggesting greater uncertainty in location and a wider activity distribution representing this location. This was a consistent observation across sessions. Error bars denote $95 \%$ confidence intervals.

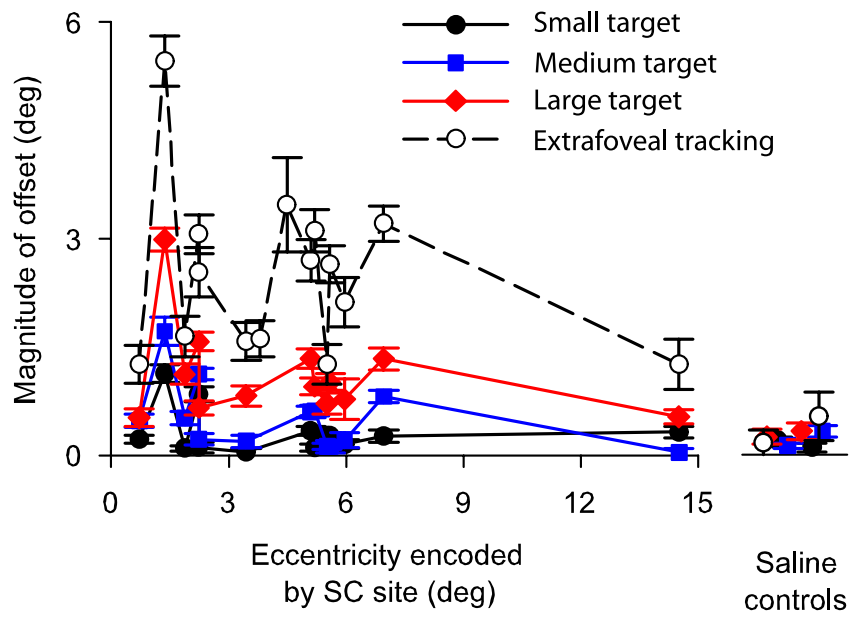

Figure 7. Summary of the effects of SC inactivation on the tracking of different target sizes. The plot shows the magnitude of the inactivation-induced offset in eye position during tracking as a function of the eccentricity encoded by the inactivated SC site as well as the size of the tracked target. Consistent with the sample experiment of Figure 6, the magnitude of the offset systematically increased as we went from the small target toward larger ones, culminating in extrafoveal tracking having the largest offsets. However, the slope of this increase was highest for parafoveal sites when compared with the most central and most eccentric experiments. The increase in offset magnitude with target size did not happen for our saline control experiments. Error bars denote $95 \%$ confidence intervals.

ing condition. This is consistent with and replicates previous results with similar visual stimuli (Basso et al., 2000). However, this observation still does not fully account for our observed ipsilesional eye-position offsets. Specifically, our offsets were largest for extrafoveal tracking, although this condition showed the least obvious changes in smooth pursuit initiation. Second, we found that ipsilesional pursuit initiation was often decreased for the small, medium, and large target conditions. If our results were explained by open-loop pursuit gain changes, then large ipsilesional eye-position offsets would require increases, not decreases, in the gain for ipsilesional pursuit.

\section{A model that can explain the stable offsets in eye position caused by inactivation}

To understand how inactivation of the rostral SC caused constant offsets in eye position, we developed a simple data-driven model explaining our results. In this model, we used recording data from the extrafoveal tracking condition, which we collected before performing the inactivation experiments to motivate these experiments, to estimate the spatial extent of the population of active neurons in the SC during this task. We then used our inactivation-induced effects on visually guided saccade latencies to estimate the spatial spread and extent of neuronal inactivation caused by muscimol injection. Our major prediction from the model is that stability of gaze, in the form of the lack of corrective movements away from the current position, is achieved through a balance of activity across the two SCs, not through the need for a special motor command from the rostral SC preventing saccades.

To estimate the neuronal distribution of SC activity during sustained extrafoveal tracking, we relied on the fact that our 117 recorded neurons during this task were from sites that spanned a wide range of eccentricities in the SC, and that these neurons were modulated by the inferred movement goal and not the visual stimuli (Fig. 2). We used these neurons to describe the spatial extent of SC activity during extrafoveal tracking by computing 

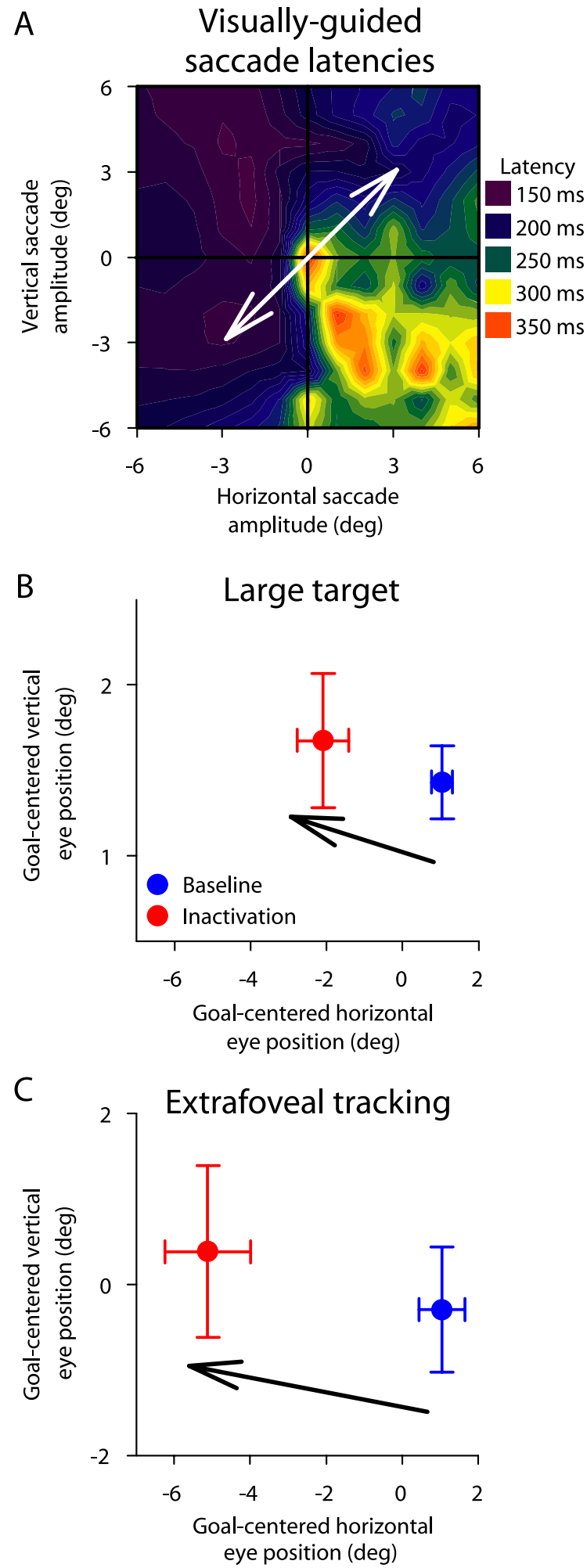

Figure 8. The direction of the eye-position offset depended on the inactivated SC site and not the tracking direction. $\boldsymbol{A}$, For one experiment, we inactivated neurons representing lower right quadrant locations. This is confirmed by observing that visually guided saccades to the lower right quadrant had longer latencies than other saccades. In addition to our typical choice of tracking direction (crossing the inactivated region), we asked our monkey in this session to also track along an orthogonal axis (white arrow). This meant that the tracking direction (as well as the peripheral bars in extrafoveal tracking) occupied locations that were not impaired by inactivation. $B, C$, For the two targets tested, the inactivation-induced offset was toward the top left quadrant relative to baseline. Moreover, the magnitude of the offset was larger for extrafoveal tracking than it was for the large target. Both of these observations are consistent with our previous results from all experiments. Error bars denote SD. the response of each neuron when the eye was aligned with the movement goal, and then binning these responses according to the preferred eccentricity of each neuron. This produced a distribution like that in Figure 9A (top), which could be described well by a Gaussian expression of the form of Equation 1 (see Materials and Methods). After normalization, we assumed circular symmetry and obtained a two-dimensional version of this function characterizing the neuron-based spatial extent of activity describing the goal location in the extrafoveal tracking task. This function is shown in Figure 9A (bottom) in SC anatomical coordinates (Eq. 2) to facilitate visualization, and its size supports our implicit hypothesis in Figures 6 and 7 that the extrafoveal tracking task recruited the activity of a significant proportion of SC neurons, including ones outside the foveal zone.

To simulate the effects of inactivation, we generated a suppression function with which the above activity profile was weighted. We assumed that the locations for which visually guided saccade latencies were largest after muscimol injections corresponded to the locations for which SC neurons were most inhibited. Thus, our suppression function was obtained by inverting and normalizing the map of visually guided saccade latencies that we measured after inactivation. This is illustrated in Figure $9 B$ for a sample experiment. As can be seen, the transformation of the data from visual coordinates into SC anatomical coordinates magnified foveal and parafoveal locations (because of the logarithmic function of Eq. 2), and therefore magnified the visualized effect of inactivation on SC neurons. This magnification was also somewhat increased by our extrapolation of the data in Figure $9 B$ (top) to fill an entire region of $-20-20^{\circ}$ in the horizontal and vertical directions in the bottom representation (see Materials and Methods). As we describe below, such extrapolation was of little consequence on the main conceptual conclusions of the model because the bulk of neuronal activity in our task was rostral (Fig. 9A).

According to our model, inactivation (i.e., multiplication of the two surfaces in Fig. 9) caused an imbalance of activity representing goal location across the two SCs, although the movement goal and the eye positions were physically aligned (Fig. 10A). Moreover, this imbalance was such that the readout of the center of mass of activity across the two SCs indicated that the target was more ipsilateral to the inactivated site than it really was. That is, with the eye physically aligned with the goal, the center of mass of the two SC maps after applying the suppression function was deviated toward the unaffected side, representing ipsilesional goal locations. This imbalance, therefore, explains the emergence of an offset in eye position and its direction.

To account for the size and constancy of the observed offsets, our model incorporates the fact that for any eye-position offset, visual feedback of the stimulus changes its retinotopic location (and that of the inferred goal) and its associated representation within the map of the SC; the center of the distribution in Figure $9 A$ would shift away from 0 with goal locations not aligned with gaze. For the sample experiment of Figure $10 \mathrm{~A}$, there existed an eye-position offset for which the new retinotopic goal location resulted in an SC population profile whose center of mass, after applying the effects of the suppression function, was zero (Fig. $10 B$ ). Thus, there was an eye-position offset for which SC activity was balanced, erroneously signaling to downstream structures (reading out target location) that the eye was on its intended goal. In this example experiment, the offset predicted by the model was $-2.6^{\circ}, 0.85^{\circ}(x, y)$, which compares favorably with the experimental values of $-2.7^{\circ}, 0.3^{\circ}$. This similarity between the model predictions and the experimental results held for all of our other 
experiments (Fig. 11), supporting the idea that it is the balance of activity across the two SCs that prevents corrective saccades, and explaining why we observed stable offsets in eye position after rostral SC inactivation rather than irrepressible saccades.

Finally, it should be noted that our model predicts different sized offsets for different sized populations of normally active neurons and a given inactivation pattern. This is consistent with our previous observations made in Figures 6 and 7, as well as those made in relation to the initial offsets at trial onset in the extrafoveal tracking condition.

\section{Discussion}

Our results demonstrate that SC activity provides a representation of behaviorally relevant goal locations in its topographic map. By reversibly inactivating some of the neurons that were normally active in our task, we showed that SC activity plays a causal role in defining where to look. Crucially, our results could not be explained by a consistent inability to either make or suppress eye movements, as might be expected from the classic view of the role of the SC (Robinson, 1972; Schiller and Stryker, 1972; Wurtz and Albano, 1980; Lee et al., 1988; Munoz and Guitton, 1991; Wurtz and Optican, 1994; Moschovakis, 1996; Hall and Moschovakis, 2004; Munoz and Wurtz, 1993a,b, 1995a,b; Sparks, 1978, 1986). Moreover, we highlighted the flexibility of the representation of goal locations in the SC by demonstrating how it varies with behavioral context, even for the same type of motor output. That is, for the same set of inactivated neurons and for the same tracking behavior, inactivation had either minimal or significant effects depending on the tracked target and its associated spatial uncertainty. These results lend strong support to the hypothesis that the SC encodes goal locations, in addition to its well-known contribution to saccade generation through saccade-related bursts (Robinson, 1972; Schiller and Stryker, 1972; Wurtz and Albano, 1980; Sparks, 1986).

\section{The goal location interpretation}

The SC is often described as issuing saccade and fixation motor commands from two putative "zones" in its caudal and rostral portions, respectively (Munoz and Guitton, 1991; Munoz and Wurtz, 1993a,b). However, attributing specific motor roles to SC neurons has proven to be problematic. For example, the so-called "fixation" neurons exhibit saccade-related bursts, and often for fairly large saccades (Munoz and Wurtz, 1993a; Krauzlis et al., 2000). Moreover, SC neurons that have been shown to possess excitatory connections to OPNs, which allow saccades by turning off their discharge (Keller, 1974; Evinger et al., 1982; Keller and Edelman, 1994; Everling et al., 1998), appear to be saccaderelated rather than fixation-related. That is, they increase their discharge at saccade onset instead of decreasing it (Gandhi and Keller, 1997). Finally, fixation-related neurons also exhibit smooth-pursuit-related activity (Basso et al., 2000; Krauzlis et al., 1997, 2000). Our results add to this growing list of evidence the observation that rostral SC inactivation did not give rise to impaired fixation and "irrepressible saccades." Instead, the eyeposition offsets that we observed were explained by a simple model in which inactivation caused a biased estimate of goal location. These results, although not designed to directly probe smooth pursuit initiation, support and extend similar suggestions made in an earlier study (Basso et al., 2000).

Our results also demonstrate the independence of the goal location representation from visual stimulation. This may be viewed as similar to the independence of saccade-related SC activity from visual stimuli. For example, humans and monkeys can voluntarily make saccades to a location opposite that of a visual stimulus (Everling et al., 1999). During this "antisaccade" behavior, saccade-related activity in the SC reflects the location of the invisible saccade endpoint (Everling et al., 1999). However, our causal inactivation effects demonstrated activity for the invisible movement goal that is not directly related to generating saccades. Similarly, Edelman and Goldberg (2003) asked monkeys to make memory-guided saccades to a briefly flashed spot that was either at the center or at the corner of a big peripheral square. Just as with antisaccades, SC activity showed saccade-related bursts for movements to the center of the square, despite the absence of 
A

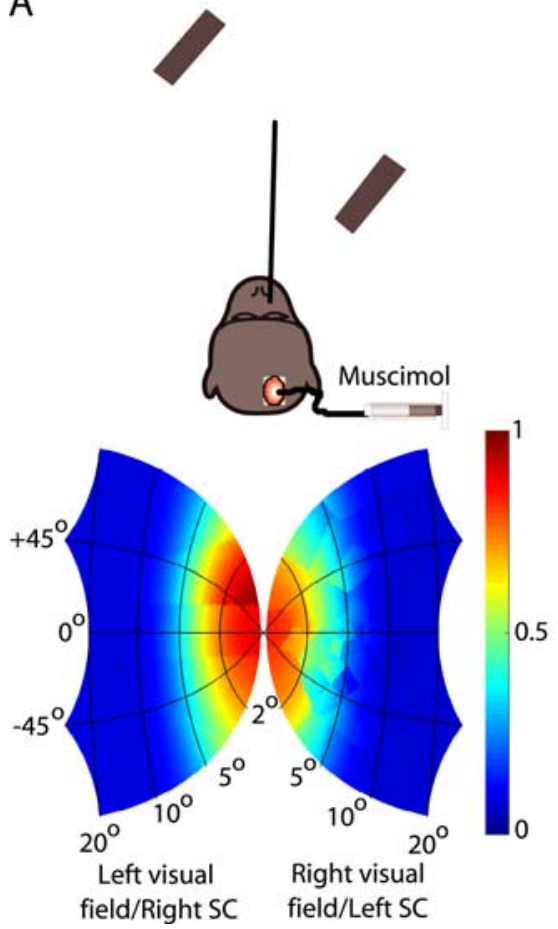

B

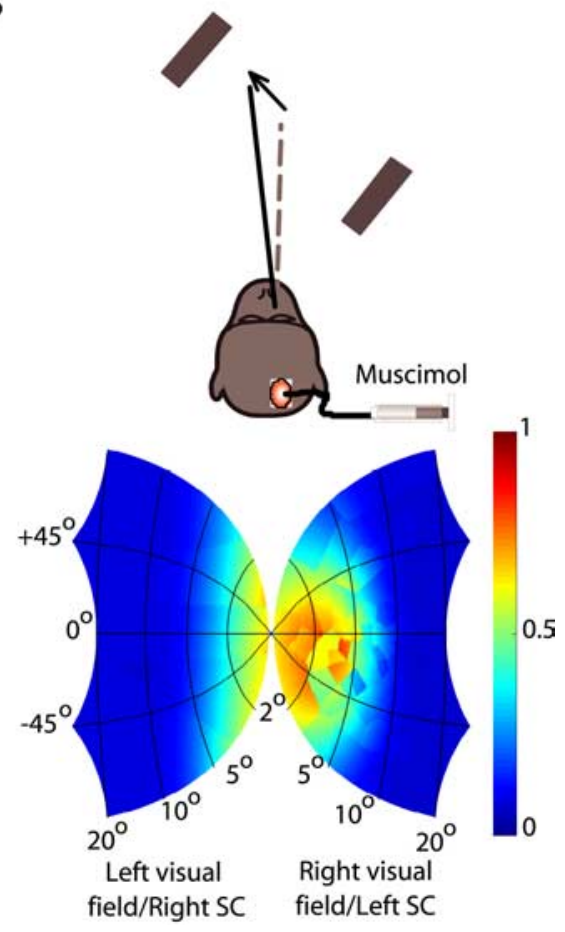

Figure 10. An explanation for the stable offsets in eye position caused by SC inactivation. $\boldsymbol{A}$, When the eye was on target, $\mathrm{SC}$ inactivation resulted in an imbalance of activity across the two maps. This is illustrated here for the sample experiment in Figure 9. Inactivation reduced the activity that was normally present in the left SC (right visual field), resulting in a shift of the center of mass of activity to the other side. $\boldsymbol{B}$, With the eye shifted away from its goal, the retinotopic location of the goal also shifted, but in the opposite direction. At a new equilibrium, the goal was at a retinotopic location that gave rise to balanced activity across the two SCS. Note how the inactivation effectively made the movement goal fall within a hole artificially created in the SC, which reduced activity in the unaffected side to reestablish balance.

A

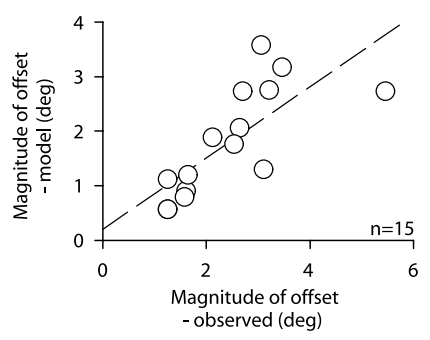

B

Angular direction difference between model offsets and experiment offsets

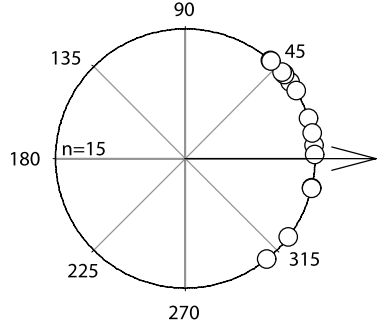

Figure 11. Our model produced results that were correlated with our data. $A$, We plotted the magnitude of the offset predicted by our model against the magnitude observed in our extrafoveal tracking experiments. Although the model undershot the data slightly, its predicted offsets were correlated with the measured ones $(r=0.75 ; p<0.01)$. The dashed line is a linear regression line. $\boldsymbol{B}$, We also looked at the difference between the direction of the offset predicted by the model and the direction of the offset observed experimentally. The model agreed with the data for all experiments. Specifically, the model predicted offset directions in the same quadrant as the data. In addition, across experiments, the average angular difference between the model and the data were not significantly different from zero ( $p=0.1, t$ test).

local visual landmarks. These previous experiments again illustrated the flexibility of saccade motor control in the SC, but they could not directly predict our results. Many neurons in the intermediate layers of the SC exhibit low-frequency activity that is not time locked to saccades (Mays and Sparks, 1980), and it is this activity that we believe contributes to representing the behavioral relevance of goal locations.

The goal location interpretation also provides an alternative explanation for the well-known finding that rostral SC inactiva-

tion increases the likelihood of unwanted saccades to abruptly appearing peripheral stimuli (Munoz and Wurtz, 1993b). In these previous experiments, irrepressible saccades were specifically triggered by and directed toward the peripheral targets, and were therefore not caused by a generalized motor impairment in fixation. Instead, the unwanted saccades occurred during paradigms involving SC activity for two competing goals: the fixated spot and the abruptly appearing peripheral stimulus. Inactivation of the neurons representing the fixated spot would be expected to bias the competition in favor of the peripheral stimulus, resulting in unwanted saccades. In our extrafoveal tracking task, the two peripheral bars also appeared abruptly, but at symmetrically opposite locations, and neither location was targeted by the inactivation. Thus, according to the goal location hypothesis, irrepressible saccades did not occur at the start of extrafoveal tracking because SC activity was not biased toward either of the two peripheral stimulus locations. During sustained extrafoveal tracking, irrepressible saccades were even less likely, because the bars did not typically recruit much SC activity during this phase of the task (Fig. 2).

\section{The role of the rostral SC}

According to the goal location interpretation of SC organization, it is not surprising that rostral SC neurons, which represent central locations, are modulated during fixation, small saccades, and smooth pursuit (Munoz and Wurtz, 1993a; Anderson et al., 1998; Krauzlis, 2003; Krauzlis et al., 1997, 2000). All of these types of motor behaviors involve foveal goal locations. However, the involvement of SC neurons in multiple types of motor outputs raises a serious issue: how does one interpret the projections from foveal and extrafoveal SC neurons to the OPN region of the brainstem? Specifically, it is known that the SC projects to OPNs and that the strength of its projections gradually decreases along the rostrocaudal extent of this structure (Gandhi and Keller, 1997; Büttner-Ennever et al., 1999; Takahashi et al., 2005). Because OPNs are tonically active during fixation and only turn off during saccades, this evidence suggests that the SC in general (at least large parts of it and not just its rostral pole) contributes to the maintenance of fixation, in addition to its contribution to the generation of saccades. How does this contribution take place? One possible mechanism, predicted by the fixation zone hypothesis (Munoz and Guitton, 1991; Munoz and Wurtz, 1993a,b), is through the maintenance of OPN activity as long as the rostral SC is active. However, this mechanism cannot explain the fact that OPNs pause for small saccades even though the rostral SC neurons connected to them increase their discharge in the same time frame.

Our results and computational model (Figs. 9-11) suggest an alternative mechanism for how OPN activity may use inputs from the SC to maintain fixation or trigger saccades. Specifically, the contribution of the SC to fixation may not be in the form of a motor command to fixate, without which OPNs become silenced, but instead in the form of an indication of the current 
state of balance across the entire SC (i.e., bilaterally). In other words, the projections to OPNs may allow these neurons to detect SC imbalances, instead of whether or not the rostral SC is active. If balance is achieved, which in our inactivation experiments happens when the eye becomes sufficiently offset from its goal, OPNs do not need to turn off and trigger a corrective movement. This mechanism explains why fairly caudal neurons, that would otherwise be labeled as "saccade" neurons according to the fixation/ saccade zone hypothesis of SC organization, often project to OPNs (Gandhi and Keller, 1997). A computation of balance across the SC that relies on more than just the most rostral portions of the map would allow stability of fixation in the face of neural noise, as well as flexibility of which neurons can be recruited to support a particular behavior. In fact, our experiments with multiple target sizes suggest that neurons in slightly caudal $\mathrm{SC}$ regions (at, say, $5^{\circ}$ of eccentricity as opposed to the fovea) may be recruited to represent a foveal goal location if the spatial uncertainty of this location is sufficiently high (Fig. 6), again consistent with the idea that projections from the SC to OPNs span fairly large portions of the SC map (Gandhi and Keller, 1997; Büttner-Ennever et al., 1999). One set of interesting future experiments could be aimed at understanding the detailed computations performed by OPNs based on their inputs from the SC.

\section{Implications on the neural basis of tracking extended objects} Finally, it is interesting to consider our results in a more general light. The common motion of the two bars in our extrafoveal tracking condition may have facilitated grouping them perceptually as a single, rigid object whose center was being tracked. In everyday life, we look at visual objects that come in a variety of shapes and sizes. Although we can look at the individual features of an object, these features are unreliable proxies for the overall object, because they often disappear behind occluders or follow trajectories that do not match that of the object (Steinbach, 1976; Beutter and Stone, 2000; Hafed and Krauzlis, 2006). These ambiguities in the sensory input create the need for a representation of object location that is suitable for use by the oculomotor system (i.e., a movement goal location). Our inactivation results causally demonstrate that the SC contains such a representation of movement goal location that is distinct from motor commands: inactivation caused an offset in eye position that was not explained by deficits expected from a pure saccade-related or fixation-related motor signal in this structure. It would therefore be interesting to investigate how this representation may interact with processes of object-based perception and attention. Given that the SC sends ascending signals through the thalamus to several cortical areas (Wurtz and Albano, 1980; Sommer and Wurtz, 2002; Wurtz et al., 2005), our finding that SC activity "points" to a movement goal location, even when there are no explicit visual features, may imply that the role of spatial location in influencing object-based attentional processes (Martinez et al., 2007) can be seeded, in part, by SC activity.

\section{References}

Adler SA, Bala J, Krauzlis RJ (2002) Primacy of spatial information in guiding target selection for pursuit and saccades. J Vis 2:627-644.

Anderson RW, Keller EL, Gandhi NJ, Das S (1998) Two-dimensional saccade-related population activity in superior colliculus in monkey. J Neurophysiol 80:798-817.

Basso MA, Krauzlis RJ, Wurtz RH (2000) Activation and inactivation of rostral superior colliculus neurons during smooth-pursuit eye movements in monkeys. J Neurophysiol 84:892-908.

Beutter BR, Stone LS (2000) Motion coherence affects human perception and pursuit similarly. Vis Neurosci 17:139-153.
Brainard DH (1997) The psychophysics toolbox. Spat Vis 10:433-436.

Büttner-Ennever JA, Horn AK, Henn V, Cohen B (1999) Projections from the superior colliculus motor map to omnipause neurons in monkey. J Comp Neurol 413:55-67.

Carello CD, Krauzlis RJ (2004) Manipulating intent: evidence for a causal role of the superior colliculus in target selection. Neuron 43:575-583.

Chen LL, Goffart L, Sparks DL (2001) A simple method for constructing microinjectrodes for reversible inactivation in behaving monkeys. J Neurosci Methods 107:81-85.

de Brouwer S, Missal M, Barnes G, Lefèvre P (2002a) Quantitative analysis of catch-up saccades during sustained pursuit. J Neurophysiol 87:1772-1780.

de Brouwer S, Yuksel D, Blohm G, Missal M, Lefèvre P (2002b) What triggers catch-up saccades during visual tracking? J Neurophysiol 87:1646-1650.

Edelman JA, Goldberg ME (2003) Saccade-related activity in the primate superior colliculus depends on the presence of local landmarks at the saccade endpoint. J Neurophysiol 90:1728-1736.

Everling S, Paré M, Dorris MC, Munoz DP (1998) Comparison of the discharge characteristics of brain stem omnipause neurons and superior colliculus fixation neurons in monkey: implications for control of fixation and saccade behavior. J Neurophysiol 79:511-528.

Everling S, Dorris MC, Klein RM, Munoz DP (1999) Role of primate superior colliculus in preparation and execution of anti-saccades and prosaccades. J Neurosci 19:2740-2754.

Evinger C, Kaneko CR, Fuchs AF (1982) Activity of omnipause neurons in alert cats during saccadic eye movements and visual stimuli. J Neurophysiol 47:827-844.

Fuchs AF, Robinson DA (1966) A method for measuring horizontal and vertical eye movement chronically in the monkey. J Appl Physiol 21:1068-1070.

Gandhi NJ, Keller EL (1997) Spatial distribution and discharge characteristics of superior colliculus neurons antidromically activated from the omnipause region in monkey. J Neurophysiol 78:2221-2225.

Hafed ZM, Krauzlis RJ (2006) Ongoing eye movements constrain visual perception. Nat Neurosci 9:1449-1457.

Hall WC, Moschovakis A (2004) The superior colliculus: new approaches for studying sensorimotor integration. Boca Raton, FL: CRC.

Hikosaka O, Wurtz RH (1985a) Modification of saccadic eye movements by GABA-related substances. I. Effect of muscimol and bicuculline in monkey superior colliculus. J Neurophysiol 53:266-291.

Hikosaka O, Wurtz RH (1985b) Modification of saccadic eye movements by GABA-related substances. II. Effects of muscimol in monkey substantia nigra pars reticulata. J Neurophysiol 53:292-308.

Ilg UJ, Thier P (1999) Eye movements of rhesus monkeys directed towards imaginary targets. Vision Res 39:2143-2150.

Judge SJ, Richmond BJ, Chu FC (1980) Implantation of magnetic search coils for measurement of eye position: an improved method. Vision Res 20:535-538.

Keller EL (1974) Participation of medial pontine reticular formation in eye movement generation in monkey. J Neurophysiol 37:316-332.

Keller EL, Edelman JA (1994) Use of interrupted saccade paradigm to study spatial and temporal dynamics of saccadic burst cells in superior colliculus in monkey. J Neurophysiol 72:2754-2770.

Krauzlis R, Dill N (2002) Neural correlates of target choice for pursuit and saccades in the primate superior colliculus. Neuron 35:355-363.

Krauzlis RJ (2003) Neuronal activity in the rostral superior colliculus related to the initiation of pursuit and saccadic eye movements. J Neurosci 23:4333-4344.

Krauzlis RJ, Miles FA (1996) Release of fixation for pursuit and saccades in humans: evidence for shared inputs acting on different neural substrates. J Neurophysiol 76:2822-2833.

Krauzlis RJ, Basso MA, Wurtz RH (1997) Shared motor error for multiple eye movements. Science 276:1693-1695.

Krauzlis RJ, Basso MA, Wurtz RH (2000) Discharge properties of neurons in the rostral superior colliculus of the monkey during smooth-pursuit eye movements. J Neurophysiol 84:876-891.

Krauzlis RJ, Liston D, Carello CD (2004) Target selection and the superior colliculus: goals, choices and hypotheses. Vision Res 44:1445-1451.

Lee C, Rohrer WH, Sparks DL (1988) Population coding of saccadic eye movements by neurons in the superior colliculus. Nature 332:357-360.

Martinez A, Ramanathan DS, Foxe JJ, Javitt DC, Hillyard SA (2007) The role 
of spatial attention in the selection of real and illusory objects. J Neurosci 27:7963-7973.

Mays LE, Sparks DL (1980) Dissociation of visual and saccade-related responses in superior colliculus neurons. J Neurophysiol 43:207-232.

McPeek RM, Keller EL (2004) Deficits in saccade target selection after inactivation of superior colliculus. Nat Neurosci 7:757-763.

Moschovakis AK (1996) The superior colliculus and eye movement control. Curr Opin Neurobiol 6:811-816.

Munoz DP, Guitton D (1991) Control of orienting gaze shifts by the tectoreticulospinal system in the head-free cat. II. Sustained discharges during motor preparation and fixation. J Neurophysiol 66:1624-1641.

Munoz DP, Wurtz RH (1993a) Fixation cells in monkey superior colliculus. I. Characteristics of cell discharge. J Neurophysiol 70:559-575.

Munoz DP, Wurtz RH (1993b) Fixation cells in monkey superior colliculus. II. Reversible activation and deactivation. J Neurophysiol 70:576-589.

Munoz DP, Wurtz RH (1995a) Saccade-related activity in monkey superior colliculus. I. Characteristics of burst and buildup cells. J Neurophysiol 73:2313-2333.

Munoz DP, Wurtz RH (1995b) Saccade-related activity in monkey superior colliculus. II. Spread of activity during saccades. J Neurophysiol 73:2334-2348.

Mustari MJ, Fuchs AF (1990) Discharge patterns of neurons in the pretectal nucleus of the optic tract (NOT) in the behaving primate. J Neurophysiol 64:77-90.

Ottes FP, Van Gisbergen JA, Eggermont JJ (1986) Visuomotor fields of the superior colliculus: a quantitative model. Vision Res 26:857-873.

Pelli DG (1997) The VideoToolbox software for visual psychophysics: transforming numbers into movies. Spat Vis 10:437-442.

Quaia C, Aizawa H, Optican LM, Wurtz RH (1998) Reversible inactivation of monkey superior colliculus. II. Maps of saccadic deficits. J Neurophysiol 79:2097-2110.
Robinson DA (1972) Eye movements evoked by collicular stimulation in the alert monkey. Vision Res 12:1795-1808.

Schiff D, Cohen B, Büttner-Ennever J, Matsuo V (1990) Effects of lesions of the nucleus of the optic tract on optokinetic nystagmus and afternystagmus in the monkey. Exp Brain Res 79:225-239.

Schiller PH, Stryker M (1972) Single-unit recording and stimulation in superior colliculus of the alert rhesus monkey. J Neurophysiol 35:915-924.

Sommer MA, Wurtz RH (2002) A pathway in primate brain for internal monitoring of movements. Science 296:1480-1482.

Sparks DL (1978) Functional properties of neurons in the monkey superior colliculus: coupling of neuronal activity and saccade onset. Brain Res 156:1-16.

Sparks DL (1986) Translation of sensory signals into commands for control of saccadic eye movements: role of primate superior colliculus. Physiol Rev 66:118-171.

Steinbach MJ (1976) Pursuing the perceptual rather than the retinal stimulus. Vision Res 16:1371-1376.

Takahashi M, Sugiuchi Y, Izawa Y, Shinoda Y (2005) Synaptic inputs and their pathways from fixation and saccade zones of the superior colliculus to inhibitory burst neurons and pause neurons. Ann NY Acad Sci 1039:209-219.

Wurtz RH, Albano JE (1980) Visual-motor function of the primate superior colliculus. Annu Rev Neurosci 3:189-226.

Wurtz RH, Optican LM (1994) Superior colliculus cell types and models of saccade generation. Curr Opin Neurobiol 4:857-861.

Wurtz RH, Sommer MA, Cavanaugh J (2005) Drivers from the deep: the contribution of collicular input to thalamocortical processing. Prog Brain Res 149:207-225.

Wyatt HJ, Pola J, Fortune B, Posner M (1994) Smooth pursuit eye movements with imaginary targets defined by extrafoveal cues. Vision Res 34: 803-820. 